\title{
Efecto del empoderamiento femenino sobre la nutrición infantil en Colombia ${ }^{1}$
}

\author{
Effect of Women's Empowerment on \\ Child Nutrition in Colombia
}

Recibido: $24-01-2018$

Aprobado: $26-10-2018$

Saluma Castillo-Guerra ${ }^{2}$

Analista de Internacionalización

Universidad del Rosario, Bogotá, Colombia

salumacg@gmail.com

https://orcid.org/0000-0002-2333-7429

$1 \quad$ Este artículo es parte del resultado del trabajo de grado presentado a la Facultad de Economía y la Escuela de Gobierno de la Universidad de los Andes como requisito de grado de máster.

2 Magíster en Economía y Magíster en Políticas Públicas 


\section{Resumen}

Este artículo estima, usando variables instrumentales, el efecto causal del empoderamiento femenino sobre el estado nutricional de niños menores de 5 años de edad en Colombia. Se utilizan como instrumentos el porcentaje de mujeres elegidas en la Asamblea Departamental y en la Cámara de Representantes, ambos retardados en el tiempo. Los resultados indican que el empoderamiento femenino reduce la desnutrición aguda y la desnutrición global en 3,52 puntos porcentuales y 5,95 puntos porcentuales, respectivamente. Se recomienda revisar los lineamientos de los programas relacionados con la política pública de equidad de género y nutrición infantil del país con el fin de diseñar políticas complementarias que mientras fortalecen la autonomía femenina, redundan en beneficio para los niños.

Palabras clave: empoderamiento; mujer; nutrición infantil.

Clasificación JEL: C26, J13, J16, J18

\section{Abstract}

Using instrumental variables estimation, this paper estimates the effect of women's empowerment on the nutrition of Colombian children who are younger than five. The instruments used were the percentage of women in the Department Assembly and the House of Representatives, both instruments lagged in time. The results show that female empowerment reduces underweight and wasting by 3.52 percentage and 5.95 percentage points, respectively. These results suggest a positive association between programs related to gender equality and nutrition policy in order to design complementary policies that strengthen women's empowerment and help improve children's wellbeing as a byproduct.

Keywords: empowerment, nutrition, women and development, maternal and child health.

¿Cómo citar este artículo? / How to quote this article?

Castillo-Guerra, S. Efecto del empoderamiento femenino sobre la nutrición infantil en Colombia. Sociedad y Economía, (36), 106-122. https://doi.org/10.25100/sye.voi36.6037 


\section{Introducción}

La nutrición es fundamental en los procesos de salud y desarrollo del niño durante los primeros años. En Colombia, los estudios relacionados con los factores determinantes en este tema se han concentrado en la educación materna, la pobreza y la falta de acceso a servicios de salud, excluyendo el rol femenino en la toma de decisiones dentro del hogar. El presente trabajo tiene como objetivo, determinar el efecto causal entre el empoderamiento femenino y el estado nutricional de niños menores a 5 años de edad en Colombia. La estimación se realiza usando variables instrumentales, como método para avanzar en el problema de variable omitida ocasionado por la variable de empoderamiento e ignorado en gran parte de la literatura.

A lo largo del trabajo se entenderá empoderamiento como "la capacidad de influir y tomar decisiones con respecto a asuntos personales y domésticos" (Dyson y Moore, 1983, p. 46). Partiendo de esta definición, se construye un índice de empoderamiento femenino con base en el análisis de correspondencias múltiples que mide el poder decisivo que tiene la mujer independientemente de la autoridad que otros puedan imponer. Los datos se obtienen de la Encuesta Nacional de Demografía y Salud (ENDS) para los años 2000, 2005 y 2010.

Empoderamiento femenino es un concepto multidimensional, difícil de medir y fuertemente influenciado por el contexto y la capacidad del individuo de elección, que dificulta aislar su efecto. En vista de la posible existencia de variables omitidas que causen simultáneamente el empoderamiento femenino y la nutrición infantil, se usan dos variaciones exógenas al modelo como variables instrumentales: el porcentaje de mujeres elegidas en la Asamblea Departamental y en la Cámara de Representantes en su circunscripción departamental, ambas rezagadas en el tiempo.

Los resultados de este ejercicio econométrico muestran que el empoderamiento femenino tiene un efecto causal positivo sobre el estado de nutrición infantil. En particular, el empoderamiento femenino reduce la desnutrición aguda y global en 3,52 puntos porcentuales (pp) y 5,95 puntos porcentuales, respectivamente. A raíz de este hallazgo, se evalúa el impacto del empoderamiento sobre posibles mecanismos de acción, encontrando un efecto significativo en prácticas de higiene, cuidado y atención tales como, si la mujer recibe capacitación sobre el tema de lactancia, si lava sus manos después de limpiar al niño y si tiene la capacidad de llevar al médico al menor en caso de estar muy enfermo.

El documento se compone de seis secciones siendo esta la inicial. La segunda sección presenta la revisión de literatura y profundiza en el problema de endogeneidad. La tercera expone la metodología usada para realizar la estimación del modelo econométrico y la cuarta describe los datos utilizados. En la sección 5, se muestran los resultados, y en la sexta, se concluye.

\section{Endogeneidad, empoderamiento femenino y nutrición infantil}

El empoderamiento, al darle mayor autonomía a las mujeres para tomar decisiones relacionadas con temas de atención de la salud, condicional en sus restricciones de tiempo e ingreso y conocimientos sobre alimentación, cuidados e higiene infantil, es un beneficio que se extiende a los niños, ya que mejora su salud y estado nutricional. Smith, Ramakrishnan, Haddad, Martorell y Ndiaye (2003) confirman que la toma de decisiones de las mujeres respecto a la de sus compañeros en el sur de África, África Subsahariana y América Latina impacta la nutrición infantil porque están mejor cuidados, y proporcionan una atención de mayor calidad a sus hijos. Estos resultados son similares a los obtenidos por Schmidt (2012)3 , quien encuentra que existe una correlación positiva entre las madres que tienen mayor autoridad y la salud de sus niños.

3 Schmidt (2012) muestra que en Bangladesh existe una relación positiva y fuerte entre niños de familias donde la mujer es quien toma decisiones como necesidades diarias y compras a largo plazo y la desnutrición crónica. 
Babu, Gajanan y Sanyal (2014) consideran que la autonomía de las mujeres es menor a la del hombre, afectando el control que tienen sobre el tiempo, el ingreso de los hogares, los conocimientos, las creencias y la confianza en sí mismas. A partir de estos aspectos considerados como medios necesarios para que la mujer tenga la capacidad de tomar decisiones y actuar de manera autónoma, vincula la relación directa e indirecta, que tiene el empoderamiento femenino con el estado nutricional infantil. En la primera categoría incluye el ingreso, las condiciones laborales, las restricciones de tiempo y la autoestima ${ }^{4}$; junta en la segunda las prácticas de cuidado y el estado nutricional.

Para la mayoría de los canales mencionados se ha encontrado que el empoderamiento femenino impacta positivamente el estado de nutrición infantil por varias razones empíricamente demostradas. En primer lugar, una mujer podrá tomar decisiones más apropiadas sobre el bienestar, la salud y nutrición de sus hijos dependiendo del control que tenga sobre su remuneración salarial y/o los recursos del hogar, lo que le permitirá invertir en el cuidado del niño. Estudios como el de Khasnobis y Hazarika (2006) y Smith et al. (2003) encuentran que cuando la mujer tiene mayor control de los recursos económicos del hogar, brinda mayor atención a sus hijos. Desde esta perspectiva, el empoderamiento femenino contribuye a un mejor estado nutricional infantil a través del gasto en alimentos y necesidades del hogar por su compromiso con la alimentación y cuidado de los niños (Rogers y Youssef, 1988).

Desai y Johnson (2005), citando a Dwyer y Bruce (1988), afirman que los hogares donde las mujeres hacen parte de la fuerza laboral, el poder de decisión para trasladar al niño a que reciba atención

4 Para esta variable, la literatura no reconoce exactamente la relación entre empoderamiento y nutrición debido a que es un mecanismo difícil de medir.

5 Rogers y Youssef (1988) describen ejemplos de programas exitosos que vinculan la participación de mujeres en actividades económicas con la importancia de los cuidados y salud infantil para señalar que las actividades económicas de generación de ingresos enfocadas hacia las mujeres están vinculadas con la salud y educación nutricional de los niños. y cuidados preventivos médicos crece, destinando una amplia proporción de su salario a los gastos del menor. En esta línea, Kumar (1979) afirma que la probabilidad de que los ingresos obtenidos por las mujeres sean invertidos en alimentos y necesidades básicas del hogar es mayor que los obtenidos por los hombres; luego, el efecto en el estado nutricional infantil será mayor y positivo.

Cuanta más autonomía tenga una mujer y menos dependa de su cónyuge porque tiene una estabilidad laboral y retribución salarial, su confianza y autoestima se incrementan. Stromquist (1995) demuestra que el trabajo remunerado le otorga mayor independencia económica en relación con la pareja, y le permite actuar libremente sobre sus recursos. No obstante, para autores como Engle, Menon y Haddad (1999), la relación entre autoestima y nutrición infantil es débil por dos limitaciones: medición de la variable y limitaciones culturales para definirla.

En segundo lugar, los cuidados nutricionales de la mujer durante y después del parto afectan el estado nutricional infantil. De acuerdo con Ramakrishnan (2001), durante el embarazo, la mujer debe proporcionarle al organismo micronutrientes y requisitos de hierro como suplemento adicional a la dieta, debido a que los niveles de hemoglobina disminuyen en esta etapa. Esto evitará retrasos de crecimiento en el niño. Bloom, Wypij y Gupta (2001) encuentran que mujeres con mayor autonomía tienen una correlación positiva con los cuidados maternos durante el embarazo puesto que son más atentas con los controles prenatales y los cuidados sugeridos que mujeres con bajos niveles de autonomía.

La alimentación después del embarazo, los cuidados de higiene y preparación de alimentos que debe tener la mujer consigo misma y con el menor influyen en su capacidad de lactar (Engle, Menon y Haddad, 1999). La relación entre lactancia y autonomía depende de un efecto comunitario. Es decir, mujeres que viven junto a otras tienen mayor autonomía de dirigir las actividades del hogar y son más proclives a amamantar a sus hijos, a diferencia de aquellas que viven en ambientes 
más coaccionados (Desai y Johnson, 2005; Vaz, Pratley y Alkire, 2015).

El poder de elegir qué es lo adecuado en temas de nutrición infantil es condicional a las capacidades intelectuales y el apoyo económico para poder sostener los gastos en los que se debe incurrir. Incluso, en aspectos psicológicos como autoestima y autoconfianza, puesto que cuando una mujer decide tener un hijo, "tiene que renunciar a su autonomía, libertad personal, identidad ocupacional, capacidad para generar ingresos, y actividades sociales y de ocio en favor de cuidar al niño" (World Health Organization, 2009, p 15). Este nuevo rol implica un proceso de adaptación que, en caso de no contar con recursos psicológicos fuertes, desencadena en alteraciones anímicas posparto que afectan la lactancia materna y la capacidad para proveer atención y cuidados al menor (Scantlan y Previdelli, 2013).

Así mismo, las variables como el gasto del ingreso femenino en productos alimenticios y otros gastos no relacionados con alimentación, las habilidades de cuidado que tiene la mujer consigo misma y con otros, y el gasto de energía que realiza son consideradas por Spring (2014) como canales de empoderamiento que contribuyen a mejorar el nivel de nutrición de ella, sus hijos y otros miembros de la familia.

La discusión sobre los canales de influencia del empoderamiento femenino en la nutrición infantil pone de manifiesto que aspectos como: el cuidado, la atención, dedicación, higiene, hábitos saludables y alimenticios, derivan en mejor estado nutricional en los niños y están al tiempo correlacionadas con el empoderamiento femenino, lo que ocasiona la presencia de un potencial sesgo de endogeneidad. Una de las razones que pueden causar este problema es la omisión de variables. Por ejemplo, es posible que una mujer muy empoderada tenga aptitudes y capacidades, o un conjunto de rasgos, cualidades o circunstancias que inciden en la influencia que ejerce a la hora de tomar decisiones en el hogar y que se correlacionan con la atención y cuidados que le brinda al niño. Intentar medir estos aspectos o tenerlos en cuenta es difícil. Por tanto, es evidente que mujeres más empoderadas pueden ser distintas de mujeres con menos autonomía en cuanto a características no observadas que resultan en mejor nutrición.

Oxaal y Baden (1997) consideran que rasgos como el poder sobre la confianza que se tiene la mujer, la autoconciencia y la asertividad se relacionan con el empoderamiento. Estas características, desarrolladas por unas mujeres más que otras, les dan mayor confianza para tomar decisiones en sus vidas al identificar su fuerza y/o su poder interior. En este sentido, medidas como la imagen que tiene la mujer de sí misma y su fuerza psicológica son variables no observadas que se omiten debido a la dificultad para obtener una medición. Por definición, si una mujer reconoce las aptitudes que le dan mayor poder interior, conduciría a una mayor capacidad para cumplir objetivos y por tanto a mayor autonomía femenina para tomar decisiones adecuadas a la salud del infante.

Así mismo, no es fácil contar con datos exactos sobre la confianza y capacidad que tiene la mujer para hacer caso omiso a los efectos de la opresión interiorizada (Rowlands, 1995); y la omisión de estas variables puede sesgar la medición. En palabras de Rowlands (1995), "una mujer que es sometida a abusos violentos cuando expresa sus propias opiniones puede empezar a retenerlas, y eventualmente llegar a creer que ella no tiene opiniones propias" (p. 102). Arraigar esta conducta reduciría el empoderamiento femenino puesto que la mujer se aparta de elegir qué es lo mejor para su hijo porque está condicionada a lo que otros suponen que es y no lo que puede llegar a ser si aumentara su confianza en sus capacidades.

Para corregir el problema de endogeneidad se debe encontrar una variable instrumental. Aunque la mayoría de los estudios realizados en torno al tema incluyen el empoderamiento femenino como una variable exógena, unos pocos han usado variables instrumentales como aproximación a la inferencia causal. En estos casos, Ahmed (2006) contempló para estudios aplicados en Nigeria la prevalencia de la poligamia y la religión como instrumentos de empoderamiento, mientras que, en Senegal, uno de los instrumentos usados por Lépine y Strobl (2013) es el origen étnico de la 
madre en relación con el de la comunidad y un cambio en el clima.

Pese a que los resultados obtenidos indican una relación positiva entre empoderamiento femenino y nutrición infantil, los instrumentos utilizados por los autores mencionados pueden tener limitaciones asociadas al cumplimiento del supuesto de la restricción de exclusión. Por ejemplo, la poligamia es una política reconocida por algunas religiones como una práctica u organización social donde se le permite al hombre convivir con varias mujeres, cada una con su espacio, aunque la primera esposa conserva el privilegio. Afirmar que este instrumento no está relacionado directamente con la nutrición infantil puede controvertirse al decir que en un hogar monogámico la mujer será más activa y tendrá mayor influencia en la toma de decisiones sobre prácticas nutricionales favorables para los infantes.

Los hogares poligámicos en África son, según Wagner y Rieger (2011), dos veces más grandes que uno monogámico y por lo general viven en condiciones precarias. Un hogar con mayor número de personas y escaso de recursos afecta la posibilidad de que un menor reciba una dieta adecuada, afectando su estado nutricional. Asimismo, la religión y la etnia también pueden estar correlacionadas con la nutrición infantil puesto que en algunos cultos se tiende a indicar la forma en la que se deben preparar los alimentos, qué dar de comer a los infantes e, incluso, prohibir ciertos alimentos que garantizan los nutrientes necesarios, modificando preferencias alimenticias. También puede estar asociado con ciertas prácticas de crianza o roles de género que afecten la nutrición.

\section{Metodología}

\section{1. Índice de empoderamiento femenino}

El término empoderamiento representa un amplio rango de conceptos. Partiendo de la definición expuesta al inicio de este documento, se construye un índice de empoderamiento femenino (IEF), como herramienta para medir el grado de participación de la mujer en asuntos personales y domésticos, a partir de las preguntas disponible en la Encuesta de Demografía y Salud (ENDS) de los años 2000, 2005 y 2010, que evalúan quién toma la decisión final $^{6}$ en cinco temas: cuidados propios en salud, gastos diarios, grandes gastos en el hogar, los alimentos a cocinar y las visitas a familiares.

Debido a que se cuenta con más de dos variables cualitativas, se utiliza el método de análisis de correspondencias múltiples (ACM) propuesto por Greenacre (2008). Esta es una técnica que generaliza el análisis de correspondencias simples para un conjunto de dos o más variables categóricas relacionadas en una matriz de Burt. El objetivo de usar este método es comparar si existe asociatividad entre las respuestas de las mujeres de la muestra o si, por el contrario, son distintas entre sí.

Se obtendrá "una matriz compuesta por todas las tablas resultantes de cruzar todas las variables de interés dos a dos" (Greenacre, 2008, p. 189). Luego, se predice un vector de índices normal estándar que es la variable de empoderamiento que entra en la regresión; cada individuo de la muestra tiene un puntaje de empoderamiento, el cual indica, por el patrón del signo, si la mujer se sitúa en los niveles más altos de empoderamiento (positivo) o en los más bajos (negativo).

\subsection{Variables instrumentales, descripción y construcción}

Para corregir la posible endogeneidad del empoderamiento femenino, se consideran dos instrumentos retardados: el porcentaje de mujeres elegidas para componer la Cámara de Representantes en su circunscripción departamental y la Asamblea Departamental. Estos dos instrumentos, condicionales en las

6 Cada uno de los cinco temas se desagrega por categorías, de las cuales se tuvieron en cuenta: solo mujer, mujer y su pareja, solo la pareja y otro. De manera que se utilizan cinco preguntas categorizadas de 1-4. 
variables de control, se consideran sustitutos del empoderamiento femenino y son extraídos de la base de datos construida por Pachón y Sánchez (2016).

Dado que la base usada agrupa los datos de la ENDS en 2000, 2005 y 2010, se añaden las variables instrumentales para cada uno de estos años retardadas en el tiempo, 35 años para la base del 2005 y en 36 para las otras dos, es decir:

- Los datos del 2000 se unen con el porcentaje de mujeres elegidas en Asamblea y Cámara en 1964.

- A los datos del 2005 se les agrega el porcentaje de mujeres elegidas en Asamblea y Cámara en 1970.

- Y, a la base del 2010 se les adhiere el porcentaje de mujeres elegidas en Asamblea y Cámara en 1974.

Según Wills (2004), para el período entre 1958 y 1970, a pesar del aumento en el porcentaje de curules ocupadas por mujeres en Cámara y Asamblea, la participación no estaba articulada con la representatividad de los intereses y necesidades en torno al tema de equidad de género. $\mathrm{Al}$ usar las variables instrumentales retardadas en el tiempo, se encuentra que el promedio de mujeres elegidas es entre $5 \%$ y $11 \%$ para Asamblea y entre $3,2 \%$ y 4,6\% en Cámara (Tabla 1), lo que da muestra de una baja representatividad femenina.

De esta manera, el instrumento es clave al estar relacionado con la idea de una baja influencia política de la mujer en el pasado y en el proceso de toma de decisiones afines a temas de desarrollo social, lo que disminuye la probabilidad de que el número de mujeres elegidas en estas corporaciones tenga efecto contemporáneo sobre la oferta departamental del estado de los servicios para los niños.

Por tanto, el porcentaje de mujeres elegidas en Cámara y Asamblea rezagado está correlacionado con el empoderamiento femenino, pero no está correlacionado con el nivel de nutrición infantil, condicional en las variables independientes. Así, el estimador resultante de la variable instrumental determinará la variación en la nutrición de los niños que se atribuyen a cambios exógenos de la autonomía de la mujer.

Es importante acotar que el número de departamentos con observaciones oscila entre 16 y 24 porque durante las décadas de 1960 y 1970, no hacían parte de la división político-administrativa de Colombia, los departamentos de Amazonas, Arauca, Guainía, Guaviare, Putumayo, San Andrés y Providencia, Vaupés, y Vichada, creados en virtud del artículo 309 de la Constitución de 1991 (Constitución Política de Colombia, 1991).

\subsection{Estrategia de estimación empírica}

Para determinar el efecto del empoderamiento femenino sobre la nutrición infantil se emplea la metodología de variables instrumentales, método que ayudará a corregir el problema de endogeneidad y obtener una estimación más puntual de la relación causal. La estimación se hace de manera separada para cada uno de los tipos de desnutrición moderada: aguda, crónica y global, términos que se entenderán solamente como desnutrición aguda, crónica o global.

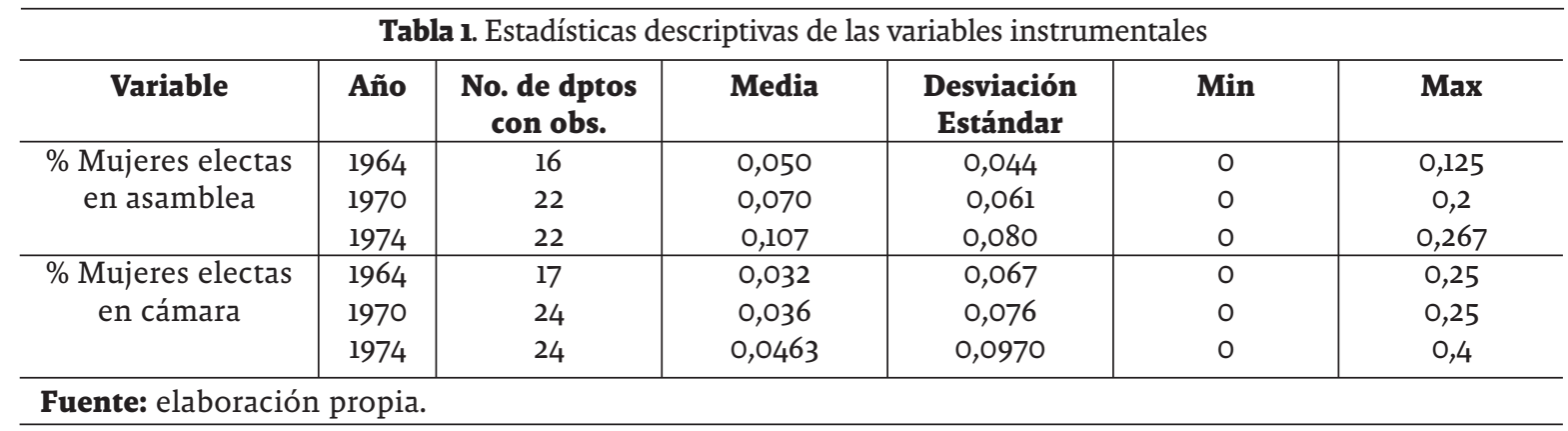


El modelo busca predecir cómo cambia la probabilidad de que un niño menor de cinco años se encuentre en un estado de desnutrición aguda, crónica o global cuando varía el índice de empoderamiento femenino en una desviación estándar. Se espera que el coeficiente que acompaña la variable de interés sea negativo, es decir, que mayor empoderamiento estaría asociado con niveles más bajos de desnutrición. El modelo formulado es el siguiente:

$y_{i d t}^{*}=\beta_{\mathrm{o}}+\beta_{1} E m p_{i d t}+\beta_{2} X^{\prime}{ }_{i t}+f_{d}+f_{t}+e_{i d t}(\mathrm{l})$

Donde:

$Y^{*}{ }_{i d t}$ es una variable definida por desnutrición crónica, desnutrición aguda o desnutrición global, las cuales indican un resultado binario que asume el valor de 1 , si un menor sufre de retraso en el crecimiento, si tiene un peso menor al que debería según su altura o si tiene un peso menor para su edad; asume el valor de cero en casos contrarios. Luego,

$$
\begin{aligned}
& y=1 \text { si } y^{*} \leq-2 \\
& y=0 \text { si } y^{*}>-2
\end{aligned}
$$

$E m p_{i d t^{\prime}}$ corresponde al índice de empoderamiento femenino estandarizado

$\beta_{1}$ es el coeficiente de interés que acompaña la variable

$X_{i t}^{\prime}$, es el vector de características observadas específicas a cada individuo

$f_{d}$, son los efectos fijos de departamento

$f_{t}$, referencia los efectos fijos de año

$e_{i d t^{\prime}}$ es el término de error de la ecuación

En la primera etapa se estima la variable endógena, que en este caso corresponde al índice de empoderamiento femenino en función del conjunto de variables instrumentales: el porcentaje de mujeres elegidas en la Asamblea Departamental $\left(A s a m_{d l}\right)$ y en la Cámara de Representantes en su circunscripción departamental $\left(\mathrm{Cam}_{d c}\right)$; las variables independientes $\left(X_{i}^{\prime}\right)$ que buscan controlar por características de la mujer, su pareja y del infante y que pueden estar correlacionadas con el estado nutricional; efectos fijos $f_{d} f_{t}$, de departamentos y temporales, respectivamente, y se derivan valores ajustados del término del error, $u_{i}$ que se incorporarán en la etapa 2. La ecuación correspondiente es:

$$
E m p_{i d t}=\propto_{0}+\propto_{1} X_{i t}^{\prime}+\propto_{2} \operatorname{Asam}_{d t}+\propto_{3} \operatorname{Cam}_{d t}+f_{d}+f_{t}+u_{i d t} \text { (2) }
$$

El coeficiente de interés en este caso es $\propto_{2}, \mathrm{y} \propto_{3}$.

\section{Datos y estadísticas descriptivas}

Para construir la base de datos, se utilizan el módulo de niños que contiene información relacionada con la talla y el peso del niño, y el módulo de los miembros de la familia de la Encuesta de Demografía y Salud (ENDS) realizada en 2000, 2005 y 2010 por Profamilia. Esta se caracteriza por recoger información sobre aspectos demográficos y de salud sexual y reproductiva en mujeres entre los 13 y 49 años de edad.

La Tabla 2 muestra las estadísticas descriptivas para una muestra de 48.586 observaciones. Reporta la media y la desviación estándar de los datos. En la Tabla 2 se puede observar que el $14 \%$ de los infantes sufre de desnutrición crónica, $1,2 \%$ de desnutrición aguda y 4,2\% de desnutrición global. Respecto a las variables instrumentales, en promedio $9 \%$ de las mujeres fueron elegidas para la Asamblea Departamental y 4,4\% para la Cámara de Representantes entre los años rezagados. El $51 \%$ de los infantes de la muestra son niños, la edad promedio es de 32 meses y $12 \%$ sufrió de enfermedad diarreica aguda durante los últimos 15 días. La edad promedio de las mujeres es de 29 años, 4 años menos que su pareja; y sus niveles de educación secundaria y educación superior, son más altos que los de su pareja. Por último, los hogares de la muestra están conformados en promedio por seis miembros y más de la mitad están ubicados en zona urbana. 


\begin{tabular}{|c|c|c|c|}
\hline Tipo de variable & Variable & Media & No. OBS. \\
\hline $\begin{array}{l}\text { Estado } \\
\text { Nutricional } \\
\text { Infantil }\end{array}$ & $\begin{array}{l}\text { Desnutrición Crónica } \\
\text { Desnutrición Aguda } \\
\text { Desnutrición global }\end{array}$ & $\begin{array}{c}0,142 \\
(0,349) \\
0,012 \\
(0,1083) \\
0,042 \\
(0,202)\end{array}$ & $\begin{array}{l}48.586 \\
48.586 \\
48.586\end{array}$ \\
\hline $\begin{array}{c}\text { Variables } \\
\text { instrumentales }\end{array}$ & $\begin{array}{l}\text { Porcentaje de mujeres electas en asamblea } \\
\text { Porcentaje de mujeres electas en cámara }\end{array}$ & $\begin{array}{c}0,090 \\
(0,074) \\
0,044 \\
(0,084)\end{array}$ & $\begin{array}{l}48.586 \\
48.586\end{array}$ \\
\hline $\begin{array}{c}\text { Características } \\
\text { del niño }\end{array}$ & $\begin{array}{c}\text { Edad (meses) } \\
\text { Sexo } \\
(\mathrm{l}=\text { niño; } 0=\text { niña }) \\
\text { Enfermedad Diarreica Aguda } \\
(\mathrm{l}=\text { si sufre; } 0=\text { no })\end{array}$ & $\begin{array}{c}32 \\
(17,08) \\
0,511 \\
(0,50) \\
0,123 \\
(0,328)\end{array}$ & $\begin{array}{l}48.586 \\
48.586 \\
48.586\end{array}$ \\
\hline $\begin{array}{c}\text { Características } \\
\text { de la mujer }\end{array}$ & $\begin{array}{c}\text { Índice de Empoderamiento } \\
\text { Nivel de Educación máxima: Básica Primaria } \\
\text { Nivel de Educación máxima: Básica Secundaria } \\
\text { Nivel de Educación máxima: Educación Superior } \\
\text { Edad (años) }\end{array}$ & $\begin{array}{c}0,0 \\
(1,0) \\
0,340 \\
(0,473) \\
0,493 \\
(0,50) \\
0,128 \\
(0,334) \\
29 \\
(6,88)\end{array}$ & $\begin{array}{l}48.586 \\
48.586 \\
48.586 \\
48.586 \\
48.586\end{array}$ \\
\hline $\begin{array}{c}\text { Características } \\
\text { de la pareja }\end{array}$ & $\begin{array}{l}\text { Nivel de Educación máxima: Básica Primaria } \\
\text { Nivel de Educación máxima: Básica Secundaria } \\
\text { Nivel de Educación máxima: Educación Superior } \\
\text { Edad (años) }\end{array}$ & $\begin{array}{c}0,384 \\
(0,486) \\
0,446 \\
(0,497) \\
0,108 \\
(0,310) \\
33 \\
(8,518)\end{array}$ & $\begin{array}{l}48.586 \\
48.586 \\
48.586 \\
48.586\end{array}$ \\
\hline $\begin{array}{l}\text { Características } \\
\text { del hogar }\end{array}$ & $\begin{array}{c}\text { Zona } \\
\text { (l=urbano; o=rural) } \\
\text { Número de miembros en el hogar } \\
\text { Índice de riqueza }\end{array}$ & $\begin{array}{c}0,628 \\
(0,483) \\
6 \\
(2,454) \\
0,00 \\
(1,00)\end{array}$ & $\begin{array}{l}48.586 \\
48.586 \\
48.586\end{array}$ \\
\hline
\end{tabular}

Desviación estándar entre paréntesis

Fuente: cálculos de la autora con base en la ENDS Colombia (2000, 2005 y 2010).

\section{Resultados y discusión}

En esta sección, se presentan los resultados de la estimación del modelo de mínimos cuadrados en dos etapas expuesto en la sección 3. Para ello, primero se estima el efecto de la variable instrumental sobre el empoderamiento femenino, y luego se usan las predicciones de dicho modelo en una segunda estimación que relaciona el efecto del empoderamiento femenino sobre la nutrición infantil. 


\subsection{Variables instrumentales}

Para controlar el posible sesgo de endogeneidad, como se mencionó previamente, se instrumenta la variable empoderamiento femenino con el número de mujeres elegidas en la Asamblea Departamental y en la Cámara de Representantes en su circunscripción departamental. Se asume que, al controlar por efectos fijos temporales y departamentales, características del niño, el hogar, la mujer y su pareja, el estado de salud de los infantes no está correlacionado con el número de mujeres elegidas en ninguna de las dos corporaciones públicas. Por consiguiente, se espera que, el número de mujeres elegidas en la Asamblea Departamental y en la Cámara de Representantes tenga efecto sobre la desnutrición solo a través del empoderamiento femenino.

La Tabla 3 presenta el R2 parcial de Shea, el R2 no centrado de la primera etapa y el $\mathrm{R} 2$ centrado y el estadístico $\mathrm{F}$ junto con su valor $p$, para una muestra de 48.586 observaciones. El R2 parcial de Shea es de 0,0054; la diferencia entre el R2 no centrado y el centrado es de 0,0004. Al ser el estadístico $\mathrm{F}$ superior a 10 se afirma que los instrumentos son conjuntamente relevantes ${ }^{7}$ para predecir el empoderamiento femenino, lo que implica que ambos instrumentos están relacionados con la variable empoderamiento.

Los resultados que se obtienen de la primera etapa (Tabla 4) indican que el porcentaje de mujeres elegidas a la Asamblea Departamental y la Cámara de Representantes favorecen el empoderamiento femenino; a mayor número de mujeres elegidas, la representación, poder e influencia de las mujeres crece, lo que resulta en aumento del empoderamiento femenino. Lo anterior sugiere que la representación de mujeres en organizaciones de representación departamental tiene influencia positiva.

\subsection{Efecto del empoderamiento femenino sobre nutrición infantil}

La Tabla 5 presenta los resultados del efecto causal del empoderamiento femenino sobre el estado nutricional infantil en

7 Los valores críticos para la prueba de instrumentos débiles de Stock y Yogo son de $19,93,11,59$ y 8,75 para $10 \%$, $15 \%$ y $20 \%$ del tamaño máximo del instrumento, respectivamente. Esta prueba se rechaza al 10\% del tamaño máximo del instrumento.

Tabla 3. Poder de predicción de los instrumentos en su conjunto

\begin{tabular}{ccccccc}
\hline Variable & Muestra & $\begin{array}{c}\text { R2 Parcial de } \\
\text { Shea }\end{array}$ & $\begin{array}{c}\text { R2 no } \\
\text { centrado }\end{array}$ & R2 centrado & Estadístico F & Valor P \\
\hline $\begin{array}{c}\text { Índice de } \\
\begin{array}{c}\text { Empoderamiento } \\
\text { Femenino }\end{array}\end{array}$ & 48.586 & 0,0054 & 0,1980 & 0,1984 & 132,39 & 0.000 \\
\hline Fuente: cálculos de la autora con base en la ENDS Colombia (2000, 2005 y 2010). & \\
\hline
\end{tabular}

Tabla 4. Resultados obtenidos en la primera etapa

\begin{tabular}{c|c}
\hline Variables Instrumentales & Empoderamiento Femenino \\
\hline Porcentaje de mujeres electas a la Asamblea & $0,153^{\star * *}$ \\
Departamental & $(0,073)$ \\
Porcentaje de mujeres electas a la Cámara de & $0,129^{* *}$ \\
Representantes & $(0,065)$ \\
Observaciones & 48.586 \\
\hline \multicolumn{2}{c}{ Errores estándares en paréntesis a nivel de clúster departamental. } \\
\multicolumn{2}{c}{${ }^{* * *}$ p $<0,01,{ }^{* *}$ p $<0,05,{ }^{*} \mathrm{p}<0,1$} \\
\hline
\end{tabular}


Colombia, medido a través de la desnutrición aguda, la desnutrición crónica y la desnutrición global. Se comparan las regresiones de mínimos cuadrados ordinarios (MCO) y las de mínimos cuadrados en dos etapas (MC2E), para indicar la dirección del sesgo.

Los resultados muestran un efecto a favor del empoderamiento femenino. Basados en la estimación de MCO se encuentra que el coeficiente que acompaña a la variable de interés se relaciona negativamente con los tres tipos de desnutrición (ver Tabla 5). Debido a que es posible que existan problemas de endogeneidad cuando se estima por MCO, se realiza el ejercicio con variables instrumentales (MC2E). El efecto estimado es en la misma dirección, pero mayor y significativo para la desnutrición aguda y la desnutrición global (ver Tabla 5).

Lo anterior significa que el efecto del empoderamiento estimado por MCO está sesgado hacia arriba, subestimando el poder de la influencia de la mujer en el hogar. Estos resultados ponen en evidencia el hecho de que mujeres más empoderadas son distintas de mujeres menos empoderadas en aspectos no observados y/o que no se pueden medir y que son relevantes para el estudio del estado nutricional infantil.
En particular, los resultados al estimar MC2E indican que el aumento en una desviación estándar en términos de empoderamiento femenino disminuye en 3,52 puntos porcentuales la probabilidad de que un niño esté en estado de desnutrición aguda, y en 5,95 pp la probabilidad de que un niño esté en estado de desnutrición global (ver Tabla 5). Es decir, existe una probabilidad menor de que los niños padezcan desnutrición con mujeres empoderadas. El hallazgo es consistente con la hipótesis inicial: mayor empoderamiento femenino presupone menores niveles de desnutrición en infantes (ceteris paribus); luego, el empoderamiento es una variable determinante del estado de nutrición infantil.

Para facilitar el entendimiento de los resultados en términos más sencillos, se separa la muestra en percentiles para determinar cuál es el cambio en el índice de empoderamiento entre mujeres. La Tabla 6 recoge en la columna 2 la variación entre los percentiles 10 y 90,25 y 50 y 50 y 75 en la distribución del índice de empoderamiento. Las tres columnas siguientes calculan el efecto del cambio entre estos percentiles en la distribución de empoderamiento sobre los tipos de desnutrición.

La diferencia en el índice de empoderamiento entre una mujer en el percentil 10 y una en el percentil 90 (ver Tabla 6) es de 2,19. El paso de una

Tabla 5. Efecto del empoderamiento femenino sobre la desnutrición aguda, desnutrición crónica y desnutrición global

\begin{tabular}{|c|c|c|c|c|c|c|c|}
\hline & \multirow[b]{2}{*}{ Variables } & \multicolumn{3}{|c|}{ MCO } & \multicolumn{3}{|c|}{ MC2E } \\
\hline & & $\begin{array}{c}\text { Desnutrición } \\
\text { aguda }\end{array}$ & $\begin{array}{c}\text { Desnutrición } \\
\text { crónica }\end{array}$ & $\begin{array}{c}\text { Desnutrición } \\
\text { global }\end{array}$ & $\begin{array}{c}\text { Desnutrición } \\
\text { aguda }\end{array}$ & $\begin{array}{c}\text { Desnutrición } \\
\text { crónica }\end{array}$ & $\begin{array}{c}\text { Desnutrición } \\
\text { global }\end{array}$ \\
\hline \multirow[t]{3}{*}{ Var de interés } & $\begin{array}{c}\text { Empoderamiento } \\
\text { Femenino }\end{array}$ & $\begin{array}{l}-0,0033^{*} \\
(0,0061)\end{array}$ & $\begin{array}{l}-0,0109^{* \star *} \\
(0,0019)\end{array}$ & $\begin{array}{l}-0,00125^{*} \\
(0,00114)\end{array}$ & $\begin{array}{l}-0,0352^{\star \star \star} \\
(0,00763)\end{array}$ & $\begin{array}{l}-0,0347 \\
(0,0237)\end{array}$ & $\begin{array}{c}-0,0595^{* \star *} \\
(0,0138)\end{array}$ \\
\hline & Constante & 0,042 & $0,295^{* *}$ & $0,0816^{* * *}$ & $0,0464^{* * *}$ & $0,298^{* * *}$ & $0,0886^{* * *}$ \\
\hline & & $(0,0080)$ & $(0,0179)$ & $(0,010)$ & $(0,00464)$ & $(0,0144)$ & $(0,00841)$ \\
\hline \multirow[t]{4}{*}{ Efectos fijos } & Controles & Sí & Sí & Sí & Sí & Sí & Sí \\
\hline & Año & Sí & Sí & Sí & Sí & Sí & Sí \\
\hline & Departamental & Sí & Sí & Sí & Sí & Sí & Sí \\
\hline & Observaciones & 48.586 & 48.586 & 48.586 & 48.586 & 48.586 & 48.586 \\
\hline
\end{tabular}

Errores estándares robustos entre paréntesis a nivel de clúster departamental. Se incluyen como controles características de niño, del hogar, la mujer y su pareja efectos fijos temporales y departamentales.

$$
{ }^{* * *} \mathrm{p}<0,01,{ }^{* *} \mathrm{p}<0,05,{ }^{*} \mathrm{p}<0,1
$$

Fuente: Cálculos de la autora con base en la ENDS Colombia (2000, 2005 y 2010). 


\begin{tabular}{cccccc}
\hline \multicolumn{5}{c}{ Tabla 6. Variación entre percentiles y efecto del empoderamiento sobre los tipos de desnutrición } \\
\hline & $\begin{array}{c}\text { Percentiles } \\
\text { (1) }\end{array}$ & $\begin{array}{c}\Delta \text { Percentil } \\
\text { (2) }\end{array}$ & $\begin{array}{c}\text { Desnutr. Aguda } \\
\text { (3) }\end{array}$ & $\begin{array}{c}\text { Desnutr. } \\
\text { Crónica } \\
\text { (4) }\end{array}$ & $\begin{array}{c}\text { Desnutr. Global } \\
\text { (5) }\end{array}$ \\
\hline Empoderamiento & $10-90$ & 2,19 & $-0,077$ & $-0,076$ & $-0,130$ \\
Femenino & $25-50$ & 0,22 & $-0,008$ & $-0,008$ & $-0,013$ \\
& $50-75$ & 0,28 & $-0,010$ & $-0,010$ & $-0,017$ \\
\hline
\end{tabular}

Fuente: cálculos de la autora con base en la ENDS Colombia (2000, 2005 y 2010).

mujer del percentil 10 (menor poder) al percentil 90 (mayor poder) genera una disminución sobre la desnutrición aguda en 7,7 pp, en la desnutrición crónica en 7,6 pp y en la desnutrición global en 13 pp. Así mismo, una mujer que se mueva en la distribución del índice del percentil 25 al 50, la desnutrición cae entre 0,08 pp y 1,3 pp.

\subsection{Canales de influencia del empoderamiento en la nutrición infantil}

Partiendo de la hipótesis de que es posible que mujeres más empoderadas que otras sean sistemáticamente diferentes debido a prácticas de cuidado, higiene y alimentación que al tiempo pueden estar correlacionadas con la variable de nutrición infantil, se estima si el empoderamiento femenino tiene efecto sobre algunas de las prácticas de cuidado asociadas a disminuir los niveles de desnutrición, con base en variables instrumentales. La ENDS realizada en Colombia en 2000, 2005 y 2010 incluye información sobre los hábitos saludables y de higiene que tiene la mujer, y la atención y los cuidados alimenticios que le brinda al niño. Basados en la revisión de literatura, se eligen las siguientes preguntas para el análisis:

- Si la mujer recibió capacitación sobre la práctica de la lactancia materna (prácticas de cuidado infantil).

- Si la mujer acostumbra a lavarse las manos inmediatamente después de que limpia a su niño (prácticas de cuidado infantil).

- Si uno de los niños llegara a estar seriamente enfermo, podría la mujer decidir por sí misma si el niño debe ser llevado a tratamiento médico (control sobre los recursos del hogar).
- Si el hijo de la mujer recibió alguna vacuna para protegerlo contra las enfermedades, incluyendo vacunas recibidas en días de campaña de vacunación (prácticas de cuidado infantil).

Los resultados (Tabla 7) sugieren que las cuatro variables nombradas anteriormente, están asociadas al empoderamiento femenino, independientemente de las características del niño, del hogar, la mujer y su pareja. La existencia de estos efectos muestra una diferencia marcada entre mujeres más empoderadas y mujeres con menor influencia en el hogar respecto a los cuidados que se deben tener con el menor para garantizarle un estado de nutrición adecuado.

Para que un niño tenga un crecimiento y desarrollo sano necesita tener una dieta balanceada que le otorgue los nutrientes necesarios durante los primeros años de vida. Una de las prácticas alimenticias esenciales para evitar problemas nutricionales es la lactancia. Los resultados obtenidos muestran que el empoderamiento femenino aumenta la probabilidad de recibir capacitación sobre la lactancia materna en 11,3 pp. Sugiere que las mujeres son conscientes de la necesidad de conocer por medio de distintas fuentes las prácticas correctas para amamantar a un bebé. Lo que resalta la importancia de intervenciones sociales que brinden información y aseguren las condiciones requeridas para que durante el embarazo la mujer adquiera una dieta variada, los suplementos nutricionales necesarios y se informe sobre el tema, conozca cuándo se debe iniciar, de qué forma y por cuánto tiempo. Esto contribuirá no solo en el peso del niño al nacer, sino que también puede ayudar a garantizar la producción de la cantidad adecuada de leche materna. 


\begin{tabular}{|c|c|c|c|c|}
\hline Variables & $\begin{array}{l}\text { Podría solo la mujer } \\
\text { decidir si llevar al } \\
\text { médico al niño }\end{array}$ & $\begin{array}{l}\text { Si la mujer lava sus } \\
\text { manos inmediata- } \\
\text { mente después de que } \\
\text { limpia a su niño }\end{array}$ & $\begin{array}{l}\text { El hijo de la mujer } \\
\text { recibió alguna vacuna } \\
\text { para protegerlo contra } \\
\text { las enfermedades }\end{array}$ & $\begin{array}{l}\text { Si la mujer recibió } \\
\text { capacitación sobre } \\
\text { lactancia materna }\end{array}$ \\
\hline $\begin{array}{l}\text { Índice de Empodera- } \\
\text { miento Femenino }\end{array}$ & $\begin{array}{l}0,0206^{* *} \\
(0,00997)\end{array}$ & $\begin{array}{l}0,121^{* * *} \\
(0,0315)\end{array}$ & $\begin{array}{l}-0,0371^{*} \\
(0,0225)\end{array}$ & $\begin{array}{c}0,113^{* *} \\
(0,0551)\end{array}$ \\
\hline Constante & $\begin{array}{c}0,848^{\star * *} \\
(0,00609)\end{array}$ & $\begin{array}{l}0,863^{\star \star *} \\
(0,0116)\end{array}$ & $\begin{array}{l}0,631^{\star \star *} \\
(0,0190)\end{array}$ & $\begin{array}{l}0,220^{\star \star *} \\
(0,0308)\end{array}$ \\
\hline $\begin{array}{c}\text { Controles } \\
\text { Efecto fijo año } \\
\text { Efecto fijo dptal }\end{array}$ & $\begin{array}{l}\text { Sí } \\
\text { Sí } \\
\text { Sí }\end{array}$ & $\begin{array}{l}\text { Sí } \\
\text { Sí } \\
\text { Sí }\end{array}$ & $\begin{array}{l}\text { Sí } \\
\text { Sí } \\
\text { Sí }\end{array}$ & $\begin{array}{l}\text { Sí } \\
\text { Sí } \\
\text { Sí }\end{array}$ \\
\hline Observaciones & 48.586 & 43.046 & 11.689 & 28.028 \\
\hline \multicolumn{5}{|c|}{$\begin{array}{l}\text { Errores estándares robustos entre paréntesis a nivel de clúster departamental. Se incluyen efectos fijos temporales y } \\
\text { departamentales. Y como controles características de niño, del hogar, la mujer y su pareja. } \\
\qquad{ }^{* *} \mathrm{p}<0,01,{ }^{* *} \mathrm{p}<0,05,{ }^{*} \mathrm{p}<0,1\end{array}$} \\
\hline
\end{tabular}

Otro canal afectado positivamente por el empoderamiento femenino es, si la mujer lava sus manos después de limpiar al niño, práctica relacionada con la preparación de alimentos y lactancia. El empoderamiento femenino aumenta la probabilidad en 12,1 pp y respalda el cuidado que tienen las mujeres con la higiene para evitar situaciones de riesgo nutricional que pueden terminar en episodios de diarreas en los infantes.

En situaciones extremas es decisivo el poder de acción de la mujer para preservar la vida del niño. El efecto del empoderamiento femenino aumenta la probabilidad en 2,06 pp, si decide llevarlo al médico en caso de estar gravemente enfermo. Este resultado es importante y cobra relevancia al momento de pensar en recomendaciones de política pues implica evaluar quién es el encargado del cuidado del menor, si esta persona tiene control sobre recursos económicos y donde se cuida, en vista que la participación de la mujer (madre) socialmente va más allá del hogar.

Al medir el efecto del empoderamiento sobre la vacunación, se obtiene que hay una relación negativa. Se esperaría que la vacunación, como una estrategia para hacer frente al contagio de enfermedades y estimular el sistema inmune de los infantes, estuviera influenciada directamente con la capacidad de la mujer para tomar decisiones dentro del hogar. No es fácil explicar a qué puede obedecer este resultado. Si bien, investigaciones recientes realizadas por MacDonald y Sage (2015) consideran que existen factores contextuales, personales y relacionados directamente con la vacunación que causan inseguridad a la hora de tomar la decisión de vacunarse o vacunar a sus hijos, también es posible pensar que las mujeres más empoderadas en comunidades pobres, dependiendo del nivel máximo de educación y sus ingresos, podrían estar en mejor capacidad de controlar los aspectos culturales que podrían impedir la vacunación en su niño.

Ahora, si se tiene en cuenta que existe un plan de vacunación obligatorio para los menores durante sus primeros años de vida, es posible que las personas duden de la vacunación porque no confíen en la eficacia y seguridad de las vacunas y/o el sistema de salud. No obstante, debido a que no se cuenta con datos para controlar estos aspectos, se recomienda en investigaciones futuras estudiar más a fondo la relación entre 
el empoderamiento femenino y la vacunación, controlando por variables que tengan influencia sobre la vacilación de la vacunación en infantes.

Aunque el resultado del análisis de canales es mixto, estos resultados reportan diferencias entre mujeres empoderadas y mujeres con menor autonomía, que marcan una diferencia significativa en el estado nutricional infantil, lo que permite considerar al empoderamiento femenino como una variable determinante de la nutrición infantil. Los resultados indican que el empoderamiento femenino tiene efecto sobre prácticas de higiene y cuidado, sin embargo, sería arriesgado decir que existe un efecto total a favor de esta variable debido a la inexistencia de efectos positivos sobre la vacunación.

\section{Conclusiones y recomendaciones}

Este artículo analizó si el empoderamiento femenino medido a través de la influencia de la mujer en la toma de decisiones relacionadas con temas domésticos y personales en el hogar tiene efecto causal sobre el estado nutricional de los niños menores de 5 años en Colombia. Los resultados permiten afirmar que, a mayor empoderamiento, la desnutrición aguda y la desnutrición global disminuyen en 3,52 y 5,95 puntos porcentuales. En particular se encontró que el empoderamiento tiene efecto negativo sobre la desnutrición crónica pero no es significativo. Este resultado es sorprendente porque no coincide con la literatura que indica relación significativa entre estas dos variables.

Entender los canales por medio de los cuales el empoderamiento femenino tiene efecto en la nutrición infantil ayuda a diseñar políticas públicas efectivas de prevención y atención a los niños que se encuentran en riesgo de desnutrición. Los resultados en materia de los mecanismos muestran efectos positivos del empoderamiento sobre si la mujer recibió capacitación sobre lactancia materna y si acostumbra a lavarse las manos después de limpiar al niño. Esto sugiere seguir potenciando estos factores a través de la promoción y defensa de la práctica de la lactancia y la promoción de campañas que den a conocer el beneficio que tiene el lavado de manos en las mujeres para la preparación de alimentos y al alimentar al infante. Esto como medidas que conducen a mejores resultados de salud y provienen de comportamientos cotidianos que las mujeres deben tener en cuenta para el cuidado del niño.

Resultados adicionales indican también un efecto positivo del empoderamiento sobre si la mujer toma decisiones para llevar al niño al médico en caso de estar enfermo. Este es un resultado esperado debido a que el canal es una situación donde la mujer puede tener autonomía o no para decidir. En términos de política implica pensar en la capacidad de respuesta del cuidador principal del infante, a quienes se les delega la responsabilidad de cuidado cuando los padres se encuentra por fuera del hogar. De manera que el cuidador principal debe conocer circunstancias especiales que le ayuden a identificar cuándo debe trasladar a los niños a organismos de salud ante una emergencia.

En este sentido, teniendo en cuenta que existe una serie de factores relevantes que potencializan el efecto del empoderamiento femenino sobre la nutrición infantil es recomendable revisar los lineamientos de los programas relacionados con la política de equidad de género del país y nutrición infantil. Esto con el fin de diseñar políticas complementarias que mientras fortalecen la autonomía femenina redundan en beneficio para el niño.

Una forma de fortalecer el empoderamiento femenino es por medio del aumento de la participación laboral y disminución de la discriminación salarial en el mercado laboral. Esto contribuirá a fortalecer la autonomía económica en las mujeres, lo cual beneficia el nivel de calidad de vida de ella y su familia. Así como también incentivando a las mujeres representantes en esferas políticas a participar en la creación de iniciativas y proyectos que promuevan la medición del impacto del trabajo doméstico y reproductivo y contribuyan al aumento del 
capital social mejorando el bienestar general de la sociedad.

Otro aspecto importante y que se debe incluir como parte de los ejes de la política de equidad de género, pues puede tener repercusiones en la nutrición infantil, es evaluar los roles de los miembros de la familia en la provisión del cuidado y atención. Si bien este es un punto susceptible de trabajar, se recomienda realizar un estudio que permita diagnosticar cuáles son las narrativas que se cuentan hombres y mujeres sobre su rol en el hogar. Para que, a partir de este, se pueda:

1. Identificar diferencias dialécticas generalizadas en las familias para obtener explicaciones culturales de los comportamientos y poder conservar perspectivas tradicionales positivas y adaptar las prácticas que puedan requerir renegociación de estructuras mentales.

2. Evaluar la importancia del control de los recursos familiares para el cuidado y la nutrición infantil junto a las practicas y los roles de los miembros de la familia.

3. Realizar pequeñas intervenciones de ensayo y error que permitan conocer cuáles son los puntos de contacto que el gobierno -e incluso empresas privadas- puede intervenir para transformar los roles de género e influir en las practicas cuidado y atención nutricional.

4. Identificar medios de influencia públicos y privados para incentivar las discusiones de roles dentro y fuera del hogar. Esto con el fin de llegar a que las futuras generaciones tomen decisiones y repartan equitativamente entre el hombre y la mujer las tareas en el hogar.

El gobierno ha enfocado sus esfuerzos en reducir las cifras de desnutrición. Sin embargo, el tema de empoderamiento femenino, el cual ha orientado a las mujeres a participar activamente en la vida económica y laboral, ha generado una brecha importante entre el tiempo dedicado al cuidado infantil desde los primeros años de vida y el tiempo dedicado a su interacción en distintos niveles de la sociedad civil. Lograr disminuir la desnutrición en Colombia implica no solo evaluar las practicas de cuidado y distribución de los recursos del hogar desde una perspectiva integrada, monitoreo, atención y tiempos de respuesta oportunos para dirigir las intervenciones a áreas y personal que lo requiera, también garantizar igualdad de condiciones a hombres y mujeres en temas que conciernen a la responsabilidad de las labores del hogar y cuidado infantil.

\section{Referencias}

Ahmed, M. (2006). Intra-household Bargaining and Investment in Child Health. Recuperado de http:// uaps2007.princeton.edu/papers/70158

Babu, S., Gajanan, S. N. \& Sanyal, P. (2014). Predicting child nutritional status using related socioeconomic variables - application of discriminant function analysis. En S. Babu, S. N. Gajanan \& P. Sanyal (Eds), Food security, poverty and nutrition policy analysis: Statistical methods and applications (pp. 201-228). San Diego, US: Academic Press.

Bloom, S., Wypij, D. \& Gupta, M. (2001). Dimensions of Women's Autonomy and the Influence on Maternal Health Care Utilization in a North Indian City. Demography, 38(1), 67-78.

Constitución Política de Colombia. (1991). Articulo 309. [Titulo 11]. Recuperado de http://www. constitucioncolombia.com/titulo-11/capitulo-2/articulo-309

Desai, S. \& Johnson, K. (2005). Women's decision making and child health: familial and social hierarchies. En S. Kishor (Ed.), A Focus on Gender Collected Papers on Gender Using DHS Data (pp. 55-68). Calverton, Maryland, USA: ORC Macro. 
Dwyer, D. \& Bruce, J. (1988). A home divided: Women and income in the third world. Stanford, California, US: Stanford University Press.

Dyson, T. \& Moore, M. (1983). On Kinship Structure, Female Autonomy, and Demographic Behavior in India. Population and Development Review, 9(1), 35-60.

Engle, P., Menon, P. \& Haddad, L. (1999). Care and Nutrition: Concepts and Measurement. World Development, 27(8), 1309-1337.

Greenacre, M. (2008). La práctica del Análisis de Correspondencias. Recuperado de http://www.fbbva. es/TLFU/dat/greenacre_apD.pdf

Khasnobis, B.\& Hazarika, G. (2006). Women's Status and Children's Food Security in Pakistan. Recuperado de https://www.wider.unu.edu/publication/womens-status-and-childrens-food-security-pakistan

Kumar, S. (1979). Impact of Subsidized Rice on Food Consumption and Nutrition in Kerala. Recuperado de http://www.ifpri.org/publication/impact-subsidized-rice-food-consumption-and-nutrition-kerala

Lépine, A. \& Strobl, E. (2013). The effect of women's bargaining power on child nutrition in rural Senegal. World Development, 45(2), 17-30.

MacDonald, N. \& SAGE. (2015). Vaccine hesitancy: Definition, scope and determinants. Vaccine, 33(34), 4161-4164.

Oxaal, Z. \& Baden, S. (1997). Gender and empowerment: definitions, approaches and implications for policy. Report No. 40. London, UK: Bridge Deveploment Gender.

Pachón, M. \& Sanchez, F. (2016). Effect of Institutional Reforms in Women's Representation in Colombia, 1960- 2014. En prensa.

Ramakrishnan, U. (2001). Functional consequences of nutritional anemia during pregnancy and early childhood. En U. Ramakrishnan (Ed.), Nutritional Anemias (pp. 43-68). Boca Raton, CA, USA: CRC Press.

Rogers, B. \& Youssef, N. (1988). The importance of women's involvement in economic activities in the improvement of child nutrition and health. Food and Nutrition Bulletin, 10(3), 33-41.

Rowlands, J. (1995). Empowerment examined. Development in Practice, 5(2),101-107.

Scantlan, J. \& Previdelli, A. (2013). Women's Empowerment and Childhood Malnutrition in Timor-Leste: A Mixed-methods Study. Portland, Oregon, US: Mercy Corps.

Schmidt, E. (2012). The Effect of Women's Intrahousehold Bargaining Power on Child Health Outcomes in Bangladesh. Undergraduate Economic Review, 9(1), Artículo 4. Recuperado de http:// digitalcommons.iwu.edu/uer/vol9/issı/4

Smith, L., Ramakrishnan, U., Haddad, L., Martorell, R. \& Ndiaye, A. (2003). The importance of women's status for child nutrition in developing countries. Recuperado de https://ageconsearch.umn.edu/ bitstream/16526/1/rro30131.pdf 
SPRING. (2014). Entendiendo la ruta del empoderamiento de las mujeres. Informe técnico $N .^{\circ}$ 4. Serie de informes sobre mejora de la nutrición a través de la agricultura. Arlington, VA, US: USAID/ Fortalecimiento de alianzas, resultados e innovaciones en la nutrición a nivel global (SPRING).

Stromquist, N. (1995). The theoretical and practical bases for empowerment. En C. Medel (Ed.), Women, Education and Empowerment: Pathways towards Autonomy (pp. 13-23). Hamburg, Alemania: UNESCO.

Vaz, A., Pratley, P. \& Alkire, S. (2015). Measuring Women's Autonomy in Chad and its Associations with Breastfeeding Practices Using the Relative Autonomy Index. Recuperado de https://ophi.org.uk/ measuring-womens-autonomy-in-chad-and-its-associations-with-breastfeeding-practices-usingthe-relative-autonomy-index/

Wagner, N. \& Rieger, M. (2011). Polygamy and Child Health. Do babies get sick if daddy has many wives? Recuperado de http://www.natascha-wagner.com/uploads/9/o/1/5/9015445/polygamy_ and_child_health.pdf

Wills, M. (2004). Las trayectorias femeninas y feministas hacia lo público en Colombia (1970-2000) ¿Inclusión sin representación? (tesis doctoral). The University of Texas, Austin, Texas, Estados Unidos.

World Health Organization. (2009). Mental health aspects of women's reproductive health: a global review of the literature. Recuperado de http://www.who.int/reproductivehealth/publications/ general/9789241563567/en/ 